\title{
Cuantificación de ivermectina eliminada en materia fecal de novillos tratados
}

\section{Ivermectin eliminated in heifers treated stool's quantification}

Fecha de recepción: 13 de noviembre de 2014 Fecha de aceptación: 26 de diciembre de 2014
Jenny Carolina Moreno-Morales', Roy José Andrade-Becerra², Martín Orlando Pulido-Medellín ${ }^{3}$

\section{Resumen}

El objetivo del presente estudio fue cuantificar la ivermectina eliminada en la materia fecal del ganado bovino, la cual fue detectada mediante la técnica de Cromatografía Líquida de Alta Resolución (CLAR), en la granja experimental Tunguavita, en la vereda Salitre de la ciudad de Paipa (Boyacá). Se experimentó en 10 novillas de diferentes razas y pesos con ivermectina comercial al $1 \%$ en una dosis de $0,2 \mathrm{mg} / \mathrm{kg}$ de peso vivo, por vía subcutánea en una sola aplicación. Se observó que al segundo día de haber aplicado el antiparasitario se obtuvo la concentración más alta (87.6\%), y a los 20 días, la más baja $(0,17 \%)$; es decir, la concentración de ivermectina fue disminuyendo, aproximadamente, en 0.0535 ppm por día. No se encontraron diferencias significativas entre razas y pesos de los animales. Los resultados de este estudio muestran la presencia de ivermectina en las heces del ganado bovino, presencia que genera un impacto ambiental negativo frente a la entomofauna coprófaga, encargada de la degradación de la materia fecal, ya que las diferentes concentraciones de ivermectina continúan con su función insecticida al ser eliminadas en la materia fecal.

De otro lado, se determinó que cierta parte del desparasitante está siendo asimilado por diferentes órganos internos del individuo, por lo tanto, no se eliminan en su totalidad.

Palabras clave: Ivermectina, Lactonas Macrocíclicas, Desparasitantes, Ivermectina en heces bovinas, Cromatografía, Bovino, Entomofauna.

1 M. Sc. Profesional independiente.

2 Ph.D. Universidad Pedagógica y Tecnológica de Colombia (Tunja, Colombia). roy.andrade@uptc.edu.co.

3 M.Sc. Universidad Pedagógica y Tecnológica de Colombia (Tunja, Colombia). martin.pulido@uptc.edu.co. 


\begin{abstract}
This study objective was to quantify the amount of ivermectin eliminated in the cattle feces, detected by the high performance liquid chromatography technique (HPLC), at the experimental farm Tunguavita in the Paipa city' Salitre townlet (Boyacá). It was experimented in 10 heifers of different breeds and weights with (the injection of) commercial Ivermectin at $1 \%$ in a $0.2 \mathrm{mg} / \mathrm{kg}$ dose of alive body weight, subcutaneously in a single application. It was noted that at the second day to have applied the antiparasite, was obtained the highest corresponding concentration at $87.6 \%$, and the lowest at 20 days after treatment, with a $0.17 \%$ concentration. That is, the Ivermectin concentration was decreasing by approximately $0.0535 \mathrm{ppm}$ per day. No significant differences were found between breeds and weights of animals. This study results show that the presence of Ivermectin in cattle feces, has a negative environmental impact on the copografa entomofauna, responsible for the degradation of the stool, as the different concentrations of Ivermectin continue their insecticide function, when eliminated in the stool.
\end{abstract}

Fufthermore, it was determined that some part of the dewormer is being assimilated by the different individual internal organs and therefore it is not removed in its entirety.

Key Words: Ivermectin, Cattle Feces, Chromatography, Bovine, Entomofauna. 


\section{Introducción}

En Colombia, una de las principales fuentes de ingreso económico es la ganadería, y para que esta sea de alta productividad, en cualquiera de sus dos propósitos (carne o leche), requiere de técnicas agrícolas eficientes; sin embargo, por factores no controlados, el ganado puede parasitarse, y se hace necesario utilizar antiparasitarios como las ivermectinas, que son las de mayor difusión por su bajo costo y su eficiencia contra parásitos internos y externos, pero tienen el inconveniente de que mantienen su actividad biológica y su poder insecticida incluso después de ser eliminadas por la materia fecal (1).

Los últimos treinta años se han caracterizado por el desarrollo y aplicación, en distintas áreas ecológicas del mundo, de numerosas estrategias de control de endo- y ectoparásitos que afectan la producción animal; la mayoría de ellas mostraron ser altamente eficaces, prácticas y económicas para el control de parásitos, pero incapaces de prevenir o controlar la contaminación ambiental que deviene de su aplicación (antihelmínticos, acaricidas, insecticidas). Casi sin excepción, y en la medida en que los antiparasitarios fueron perdiendo eficacia, estas estrategias se hicieron menos rentables, comprometiendo, en algunos casos, la propia sustentabilidad del sistema productivo (1).

La ivermectina (IVM) es una Lactona Macrocíclica (LM) perteneciente al grupo de las avermectinas; aparece en la década de los ochenta del pasado siglo, demostrando efectividad frente a un gran número de parásitos tanto internos como externos, y permite varias formas de administración (2). Desde que se inició su comercialización, en 1981, la IVM ha sido ampliamente utilizada en explotaciones de rumiantes, porcinos y equinos, así como en animales de compañía, debido a su amplio espectro de acción (3); es endectocida eficiente en el control de endo- y ectoparásitos.

Las LM son activas para el control de nematodos y artrópodos, a dosis bajas, en la mayoría de los animales domésticos; se absorben por todas las vías, debido a su alta liposolubilidad, y se distribuyen ampliamente en los tejidos, tales como la luz in- testinal, la grasa y la piel; producen su efecto antiparasitario al incrementar la permeabilidad de la membrana celular por los iones de cloro (Cl-), con la consecuente hiperpolarización y parálisis de la musculatura faríngea y somática de los parásitos $(3,4)$. Pueden detectarse en bajas concentraciones en el plasma sanguíneo, heces y en diversos tejidos (músculo, hígado) mediante la utilización de variadas técnicas. CLAR es una de las técnicas más sensibles para la detección de fármacos, y se basa en la formación de un derivado fluorescente de la sustancia en análisis (5).

El objetivo de la presente investigación fue cuantificar, bajo la técnica de CLAR, la presencia de Lactonas Macrocíclicas eliminadas en la materia fecal del ganado bovino en diferentes tiempos postratamiento.

\section{Materiales y métodos}

El estudio inicial se realizó en la granja experimental Tunguavita, localizada en el km 4 Vía Paipa-Toca (Boyacá), vereda El Salitre (latitud 0.5 $45^{\prime}$ Norte y Longitud $73^{\circ} 45^{\prime}$ Oeste), con una altura de 2480 m.s.n.m, una temperatura promedio de $14.3^{\circ} \mathrm{C}$, humedad relativa de $78 \%$, precipitación de 737.9 mm3/año (9), y una extensión de 135 ha.

Dentro del grupo experimental se escogieron 20 novillas sin tener en cuenta la carga parasitaria, el peso corporal y la raza; se separaron en dos grupos de 10 animales cada uno: al grupo I no se le suministró tratamiento, pero se le aplicó agua destilada estéril como placebo, y el grupo II fue tratado con ivermectina comercial. Durante los 20 días del período experimental, cada grupo permaneció en potreros separados por una cerca eléctrica. La extensión del potrero era de, aproximadamente, una hectárea.

A los animales del grupo tratado se les inyectó ivermectina al $1 \%$ por vía subcutánea en una dosis de $0,2 \mathrm{mg} / \mathrm{kg}$ de peso vivo y con tiempo de retiro de 28 a 35 días postratamiento (4).

Las muestras de heces se recolectaron directamente del recto con manga de palpación nueva y desechable; la primera, antes de empezar el trata- 
miento con ivermectina (tiempo 0) y las siguientes en los días $1,2,4,6,8,12,14,16,19$ y 20 , posteriores al tratamiento.

Las 20 muestras de materia fecal, con un peso aproximado de 10 gramos cada una, se almacenaron a una temperatura de $-195,8{ }^{\circ} \mathrm{C}$, con nitrógeno líquido, hasta su análisis en el laboratorio (10).

Las muestras fueron llevadas al Laboratorio de análisis cromatográfico de Corpoica-Mosquera; cada una de ellas con 2 repeticiones, para ser agrupadas y determinar las concentraciones de ivermectina mediante el uso de un detector de fluorescencia CLAR(10) Los resultados se expresaron en partes por millón.

\section{Resultados y discusión}

Tabla I. Concentración por día de ivermectina extraída de materia fecal de ganado bovino

\begin{tabular}{|l|l|l|}
\hline DíAS & $\begin{array}{c}\text { CONCENTRACIÓN } \\
(\mathbf{p p m})\end{array}$ & $\begin{array}{c}\text { CONCENTRACIÓN } \\
\text { EN \% }\end{array}$ \\
\hline 2 & 0,8760 & 87,6 \\
\hline 3 & 0,8520 & 85,2 \\
\hline 4 & 0,7320 & 73,2 \\
\hline 6 & 0,6540 & 65,4 \\
\hline 8 & 0,5510 & 55,1 \\
\hline 12 & 0,2410 & 24,1 \\
\hline 14 & 0,1100 & 11 \\
\hline 16 & 0,0120 & 1,2 \\
\hline 19 & 0,0041 & 0,41 \\
\hline 20 & 0,0017 & 0,17 \\
\hline
\end{tabular}

En la Tabla I se observa que a medida que pasan los días después de la aplicación disminuye la concentración de ivermectina en la materia fecal $(p=$ 5.33 E-07); se aprecia que la concentración inicial $(0,8760)$ es muy alta, comparada con la concentración final $(0,0017)$, al día 20 postratamiento.

Estudios previos realizados por Iglesias (2006) muestran que las concentraciones más altas de ivermectina presentes en las heces de animales tratados por vía subcutánea se encuentran a los 3 días postratamiento (0.717 ppm), y las más bajas (0.0021 ppm) se encontraron a los 21 días postratamiento (11); así mismo, estudios realizados por Suarez (2003) determinaron que las concentraciones de IVM fecales fueron de 1150 ppm (día 3 postratamiento) y $22,8 \mathrm{ppm}$ (día 29 postratamiento) (6); muy altas comparadas con el presente estudio.

En comparación con moxidectina (LM), el valor de concentración máxima encontrado en materia fecal húmeda es de 5,2 ppm, registrado a los 6 días de haberse aplicado el desparasitante. Esto indica que se presenta un pico de concentración, ya que a los primeros días no se obtuvieron concentraciones de LM, y después del sexto día fue disminuyendo progresivamente (13).

Con respecto al coeficiente de determinación, la variabilidad de concentración está explicada en un $96.28 \%$; de acuerdo con una relación lineal con los días, las concentraciones de IVM disminuyen con el tiempo en las deposiciones fecales del ganado. La variable correspondiente a las concentraciones de IVM depende del tiempo, ya que posterior al tratamiento las concentraciones del desparasitante disminuyen a medida que aumenta el tiempo.

Las variables están fuertemente relacionadas; se disminuye la concentración de IVM en 0.0535 ppm por cada día que trascurre; es decir, en los intervalos de tiempo tomados dentro del tratamiento dejan ver la reducción antihelmíntica en una constante de acuerdo con los tiempos de toma de muestras de las heces.

Tabla II. Porcentaje de recuperación de ivermectina con la aplicación de diferentes concentraciones en las heces de ganado bovino.

\begin{tabular}{|l|l|}
\hline Concentración (ppm) & Recuperación (\%) \\
\hline 10 & 75,4 \\
\hline 20 & 83,2 \\
\hline 50 & 80,6 \\
\hline 100 & 87,7 \\
\hline 200 & 87,9 \\
\hline 400 & 90,6 \\
\hline
\end{tabular}


Aplicando la misma técnica de análisis de CLAR (10), hay una recuperación del $0.0299 \%$ por cada unidad en ppm de IVM contenida en las heces. Se determinó que a mayor cantidad de concentración aplicada, mayor porcentaje de recuperación del desparasitante; sin embargo, con la aplicación de estas diferentes concentraciones directas en la materia fecal, no se obtuvo una recuperación en su totalidad, es probable que esa cantidad se haya degradado por otros factores.

\section{Conclusiones}

La concentración inicial de IVM encontrada en la materia fecal en el segundo día postratamiento es de 0,8760 ppm, concentración muy alta comparada con la concentración final $(0,0017$ ppm), a los 20 días postratamiento.

Es importante destacar que gran parte del contenido del desparasitante aplicado se acumula en el moco y el contenido intestinal, de ahí las altas concentraciones obtenidas en la materia fecal.

Se evidencia que en el segundo día postratamiento se recuperó una concentración superior, comparada con los demás días postratamiento. Los resultados están relacionados con que cierta parte del desparasitante está siendo asimilado por diferentes agentes biológicos internos o externos del individuo.

Las concentraciones obtenidas en la materia fecal después de haber aplicado la IVM al ganado bovino indican que este antihelmíntico continúa ejerciendo su función tóxica, causando daño a la entomofauna, provocando erosión al suelo y afectando al ser humano.

Los límites máximos de residuos de IVM permitidos en bovinos, según la legislación vigente (resolución 1382 de 2013, ministerio de salud), son $0,04 \mathrm{ppm}$ en grasa y 0,1 ppm en hígado, por lo tanto, se puede concluir que las concentraciones encontradas en el estudio en la materia fecal exceden los límites establecidos.

Según Rodríguez (2010), el tiempo normal de liberación de IVM en la materia fecal corresponde a
7 días postratamiento, pero en este estudio fue de 2 días postratamiento, lo que indica que el tiempo de retención del antihelmíntico se reduce y, así mismo, se elimina en gran cantidad en las heces, por lo cual el organismo del individuo tratado no lo está asimilando en su normalidad.

\section{Referencias}

(1) Aparicio J., Paredes V., González O., Navarro O. Impacto de la ivermectina sobre el ambiente. Revista Científica La Calera. 2011; 1998-8850, Managua, Nicaragua.

(2) Chaparro E. Determinación de resistencia antihelmíntica frente a las lactonas macrocíclicas por parte de nemátodos gastrointestinales del equino mediante el test de reducción de la oviposición (fecrt) en los municipios de Aguazul, el Yopal, Maní y Paz de Ariporo, departamento del Casanare, Colombia. Facultad de Medicina Veterinaria. Universidad De La Salle. Bogotá, 2008.

(3) García B., Hernández D., Soler F., Pérez M. Empleo de ivermectina como parasiticida en ovinos: Posibles efectos tóxicos y repercusiones ambientales. Unidad de Toxicología. Rev Científica de la Universidad de Murcia. $2011 ; 23-32$.

(4) Rodríguez R., Arieta R., Pérez L., Rosado J., Ramírez G., Basto G. Uso de lactonas macrocíclicas para el control de la garrapata Rhipicephalus (Boophilus) microplus en el ganado bovino. Rev Redalyc.org 2010; 42(3): 115-123.

(5) Pincheira A. Comparación del perfil de excreción fecal de ivermectina administrada por vía subcutánea en ovinos parasitados y sin parasitismo gastrointestinal. Facultad de Medicina Veterinaria. Chillán, Chile, 2005.

(6) Suárez V., Lifschitz A., Sallovitz J., Lanusse C. Efectos de la ivermectina y doramectina de los residuos fecales en la colonización de invertebrados del estiércol de ganado. Rev Tropical 
and Subtropical Agroecosystems. La Pampa, Argentina; 2003; 127, 481-488.

(7) Lumaret J., Martínez I. Ensayo El impacto de productos veterinarios sobre insectos coprófagos: consecuencias sobre la degradación del estiércol en pastizales. Universidad Paul Valéry. Acta Zoológica Mexicana (n.s.). 200; 21(3): 137-148.

(8) Muñoz A., Angulo F., Ramírez R., Vale O., Chacín E., Simoes D. y Atencio A. Eficacia antihelmíntica de doramectina $1 \%$, ivermectina $1 \%$ y ricobendazol $15 \%$ frente a nematodos gastrointestinales en ovinos de pelo. Rev. Cient. (Maracaibo), 2008; Vol. XVIII, N. ${ }^{\circ}$ 1, 12-16.

(9) Sanabria A. Granja Experimental Tunguavita Universidad Pedagógica y Tecnológica de Colombia. Paipa Proyectos Productivos Pecuarios, 2010. Tunja, Boyacá.

(10) Navias C. Comparación del perfil de excreción fecal de moxidectina luego de la administración subcutánea en ovinos con y sin parasitismo gastrointestinal. Facultad de Medicina Veterinaria. Universidad de Concepción. Departamento de Ciencias Clínicas. Chillán, Chile, 2006.

(11) Iglesias L., Saumell C., Fernández A., Fusé L., Lifschitz A., Rodríguez E., Steffan P., Fiel C. Ef- fects of ivermectin and doramectin faecal residueson the invertebrate colonization of cattle dung. Journal of Applied Entomology. 2006; Parasitol Res 100: 93-102.

(12) Flota C., López J., Vargas M., Fajersson P., González H., Martínez I. Efecto de la ivermectina en la dinámica espacio-temporal de escarabajos estercoleros en Veracruz, México. Rev Tropical and Subtropical Agroecosystems, 2012; 227-239.

(13) Pérez R., Cabezas I., Godoy C., Rubilar L., Díaz L., Muñoz L., Arboix M., Alvinerie M. Disposición plasmática y fecal de moxidectina administrada por vía oral en caballos. Chillán, Chile. Arch. med. vet., 2001; 33(1): 0301732.

(14) Rodríguez V., Arieta R., Pérez L., Rosado J., Ramírez G., Basto G. Uso de lactonas macrocíclicas para el control de la garrapata Rhipicephalus (Boophilus) microplus en el ganado bovino. Yucatan, Mexico. Arch Med Vet. 2010; 42, 115-123.

(15) Resolución 1382/2013 de 2 de mayo. Límites máximos para residuos de medicamentos veterinarios en los alimentos de origen animal destinados al consumo humano. Ministerio de Salud y Protección Social. República de Colombia. 